Pacific Journal of Mathematic 


\section{EIGENVALUES OF THE UNITARY PART OF A MATRIX}

\section{AlFRed Horn AND ROBERT Steinberg}

1. Introduction. It is well known that every matrix $A$ (square and with complex entries) has a polar decomposition $A=P_{1} U_{1}=U_{2} P_{2}$, where $U_{i}$ are unitary and $P_{i}$ are unique positive semi-definite Hermitian matrices. If $A$ is non-singular then $U_{1}=U_{2}=U$, where $U$ is also unique. In this case we call $U$ the unitary part of $A$. The eigenvalues of $P_{1}$ are the same as those of $P_{2}$.

In [2] the following problem was solved. Given the eigenvalues of $P_{1}$, what is the exact range of variation of the eigenvalues of $A$ ? The answer shows that a knowledge of the eigenvalues of $P_{1}$ puts restrictions only on the moduli of the eigenvalues of $A$. In this paper we are going to consider the corresponding question for the unitay part $U$ of $A$. In turns out that a knowledge of the eigenvalues of $U$ restricts only the arguments of the eigenvalues of $A$.

Before stating the result, we need some definitions. An ordered pair of $n$-tuples $\left(\lambda_{i}\right),\left(\alpha_{i}\right)$ of complex numbers is said to be realizable if there exists a non-singular matrix $A$ of order $n$ with eigenvalues $\lambda_{i}$ such that the unitary part of $A$ has eigenvalues $\alpha_{i}$. If $\left(\gamma_{j}\right)$ is an $n$-tuple of complex numbers of modulus 1 , and if two of the $\gamma_{j}$ are of the form $e^{i b}, e^{i c}$ with $0<b-c<\pi$ and $0 \leqq d \leqq(b-c) / 2$, then the operation of replacing $e^{i b}$, $e^{i c}$ by $e^{i(b-a)}, e^{i(c+d)}$ is called a pinch of $\left(\gamma_{j}\right)$. In other words, a pinch of $\left(\gamma_{j}\right)$ consists in choosing two of the $\gamma_{j}$ which do not lie on the same line through 0 and turning them toward each other through equal angles.

If $\left(a_{i}\right),\left(b_{i}\right)$ are $n$-tuples of real numbers, and if $\left(a_{i}^{\prime}\right),\left(b_{i}^{\prime}\right)$ are their rearrangements in non-decreasing order, then we write $\left(a_{i}\right) \prec\left(b_{i}\right)$ when $\sum_{r}^{n} a_{i}^{\prime} \leqq \sum_{r}^{n} b_{i}^{\prime}, r=2, \cdots, n$ and $\sum_{1}^{n} a_{i}^{\prime}=\sum_{1}^{n} b_{i}^{\prime}$. It is easily seen that the conditions are equivalent to the conditions $\sum_{1}^{r} a_{i}^{\prime} \geqq \sum_{1}^{r} b_{i}^{\prime}, r=1, \cdots$, $n-1$, and $\sum_{1}^{n} a_{i}^{\prime}=\sum_{1}^{n} b_{i}^{\prime}$.

Our main theorem is the following.

THEOREM 1. Let $\left(\lambda_{i}\right),\left(\alpha_{i}\right)$ be n-tuples of complex numbers such that $\lambda_{i} \neq 0$ and $\left|\alpha_{i}\right|=1$. Then the following statements are equivalent:

(1) the pair $\left(\lambda_{i}\right),\left(\alpha_{i}\right)$ is realizable;

(2) $\left(\alpha_{i}\right)$ can be reduced to $\left(\lambda_{i}|| \lambda_{i} \mid\right)$ by a finite sequence of pinches;

(3) $\prod_{1}^{n} \alpha_{i}=\Pi_{1}^{n}\left(\lambda_{i}|| \lambda_{i} \mid\right)$, and exactly one of the following hold:

(a) there is a line through 0 containing all the $\alpha_{i}$ and $\left(\lambda_{i} /\left|\lambda_{i}\right|\right)$ is a rearrangement of $\left(\alpha_{i}\right)$;

(b) there is no line through 0 containing all $\alpha_{i}$ but there is

Received September 26, 1958. 
a closed half plane $H$ with 0 on its boundary containing all $\alpha_{i}$, and, if we choose a branch of the argument function which is continuous in $H-\{0\}$, then $\left(\arg \lambda_{i}\right) \prec\left(\arg \alpha_{i}\right)$;

(c) there is no closed half plane with 0 on its boundary which contains all $\alpha_{i}$.

The proof of Theorem 1 will be given at the end of the paper.

2. Definitions and preliminary results. Two matrices $A$ and $B$ are said to be congruent if there exists a non-singular matrix $X$ such that $B=$ $X^{*} A X$. A triangular matrix is a matrix such that all entries below the main diagonal are 0 . If $P$ is a positive definite matrix, then $P^{1 / 2}$ denotes the unique positive definite matrix whose square is $P$. We will use the symbol diag $\left(a_{1}, \cdots, a_{n}\right)$ to denote the diagonal matrix with diagonal elements $a_{1}, \cdots, a_{n}$.

LemmA 1. If $\lambda_{i} \neq 0$ and $\left|\alpha_{i}\right|=1$, then the pair $\left(\lambda_{i}\right),\left(\alpha_{i}\right)$ is realizable if and only if there exists a matrix $A$ with eigenvalues $\lambda_{i}$ which is congruent to $D=\operatorname{diag}\left(\alpha_{1}, \cdots, \alpha_{n}\right)$.

Proof. We use the fact that for any two matrices $B$ and $C, B C$ and $C B$ have the same eigenvalues. If $\left(\lambda_{i}\right),\left(\alpha_{i}\right)$ is realizable, there exists a unitary matrix $U$ with eigenvalues $\alpha_{i}$ and a positive definite matrix $P$ such that $P U$ has eigenvalues $\lambda_{i}$. Let $V$ be a unitary matrix such that $U=V^{*} D V$. Then $P U$ has the same eigenvalues as $P^{1 / 2} V^{*} D V P^{1 / 2}$, which is congruent to $D$. Conversely, if $X^{*} D X$ has eigenvalues $\lambda_{i}$, then so does $A=X X^{*} D$, and $D$ is the unitary part of $A$ since $X X^{*}$ is positive definite.

Lemma 2. If $\left(\lambda_{i}\right),\left(\alpha_{i}\right)$ is realizable and $\rho_{i}>0$ for each $i$, then $\left(\rho_{i} \lambda_{i}\right),\left(\alpha_{i}\right)$ is realizable.

Proof. Suppose $D=\operatorname{diag}\left(\alpha_{1}, \cdots, \alpha_{n}\right)$ is congruent to a matrix $A$ with eigenvalues $\lambda_{i}$. Then $A$ is congruent to a triangular matrix $B$ with diagonal elements $\lambda_{i}$. If $X=\operatorname{diag}\left(\rho_{1}^{1 / 2}, \cdots, \rho_{n}^{1 / 2}\right)$, then $X^{*} B X$ obviously has eigenvalues $\rho_{i} \lambda_{i}$ and is congruent to $D$.

Lemma 3. If $\left(\lambda_{i}\right),\left(\alpha_{i}\right)$ is realizable and $z$ is any complex number of modulus 1 , then $\left(z \lambda_{i}\right),(z \alpha)$ is realizable.

Lemma 4. If $\left(\mu_{1}, \mu_{2}\right)$ results from $\left(\lambda_{1}, \lambda_{2}\right)$ by a pinch and $T$ is a triangular matrix with diagonals elements $\lambda_{1}, \lambda_{2}$, then $T$ is congruent to a matrix with eigenvalues $\mu_{1}, \mu_{2}$.

Proof. By multiplication by a suitable constant, we may suppose 
that $\lambda_{1}=e^{i \theta}, \lambda_{2}=e^{-i \theta}$, and $\mu_{1}=e^{i \phi}, \mu_{2}=e^{-i \phi}$, where $0 \leqq \phi \leqq \theta<\pi / 2$. It suffices to find a positive matrix $P$ such that $P T$ has eigenvalues $e^{ \pm i \phi}$. Suppose

$$
T=\left(\begin{array}{cc}
e^{i \theta} & a \\
0 & e^{-i \theta}
\end{array}\right)
$$

Let

$$
P=\left(\begin{array}{ll}
x & \bar{y} \\
y & x
\end{array}\right),
$$

where $x \geqq 1,|y|^{2}=x^{2}-1$ and $y a=|a|\left(x^{2}-1\right)^{1 / 2}$. Since $P$ has determinant 1 , we need only choose $x$ so that the trace of $P T$ is $2 \cos \phi$. The trace of $P T$ is $f(x)=x e^{i \theta}+x e^{-i \theta}+y a=2 x \cos \theta+|a|\left(x^{2}-1\right)^{1 / 2}$. When $x=1$, this is $2 \cos \theta$, and for $x \geqq 1, f(x)$ increases to infinity.

Lemma 5. If $\left(\alpha_{i}\right)$ can be reduced to $\left(\lambda_{i} /\left|\lambda_{i}\right|\right)$ by a finite number of pinches, then $\left(\lambda_{i}\right),\left(\alpha_{i}\right)$ is realizable.

Proof. By Lemma 2 we may assume $\left|\lambda_{i}\right|=1$. We need only prove the following: if $\left(\lambda_{i}\right),(\alpha)$ is realizable, if $\left|\lambda_{i}\right|=1$ and if $\left(\mu_{i}\right)$ is a pinch of $\left(\lambda_{i}\right)$, then $\left(\mu_{i}\right),\left(\alpha_{i}\right)$ is realizable. We may suppose that the pinch consists in replacing $\lambda_{1}, \lambda_{2}$ by $\mu_{1}, \mu_{2}$. By hypothesis there exists a triangular matrix $A$ with eigenvalues $\lambda_{i}$ which is congruent to diag $\left(\alpha_{1}, \cdots, \alpha_{n}\right)$. By Lemma 4 there exists a two rowed non-singular matrix $Z$ such that

$$
B=Z^{*}\left(\begin{array}{cc}
\lambda_{1} & a_{12} \\
0 & \lambda_{2}
\end{array}\right) Z
$$

has eigenvalues $\mu_{1}, \mu_{2}$. Here $a_{12}$ is the $(1,2)$ entry of $A$. If we set

$$
Y=\left(\begin{array}{ll}
Z & 0 \\
0 & I
\end{array}\right)
$$

where $I$ is the identity matrix of order $n-2$, then

$$
Y^{*} A Y=\left(\begin{array}{ll}
B & C \\
0 & D
\end{array}\right),
$$

where $D$ is triangular with diagonal elements $\lambda_{3}, \cdots, \lambda_{n}$. But this last matrix obviously has eigenvalues $\left(\mu_{1}, \mu_{2}, \lambda_{3}, \cdots, \lambda_{n}\right)=\left(\mu_{1}, \cdots, \mu_{n}\right)$.

Lemma 6. If $\left(a_{1}, \cdots, a_{k}\right) \prec\left(b_{1}, \cdots, b_{k}\right)$ and $\left(c_{1}, \cdots, c_{p}\right) \prec\left(d_{1}, \cdots, d_{p}\right)$ then $\left(a_{1}, \cdots, a_{k}, c_{1}, \cdots, c_{p}\right) \prec\left(b_{1}, \cdots, b_{k}, d_{1}, \cdots, d_{p}\right)$. 
Proof. A proof is given in $[1 ; 63]$.

Lemma 7. If $A$ is a matrix such that $(A x, x) \neq 0$ and $0<$ $\arg (A x, x)<\pi$ for all $x \neq 0$, then $A$ is congruent to a unitary matrix.

Proof. Let $H=\left(A+A^{*}\right) / 2, K=\left(A-A^{*}\right) / 2 i$. Then $A=H+i K$, and $H, K$ are Hermitian. Since $(A x, x)=(H x, x)+i(K x, x)$, the hypothesis implies that $(K x, x)>0$ for all $x \neq 0$, so that $K$ is positive definite. Therefore by $[3 ; 261] H$ and $K$ are simultaneously congruent to real diagonal matrices. Hence $A=H+i K$ is congruent to a diagonal unitary matrix.

Lemma 8. If $A$ is congruent to a unitary matrix $U$ with eigenvalues $\alpha_{i}$, and if $0<\arg \alpha_{1}<\cdots<\arg \alpha_{n}<\pi$, then $(A x, x) \neq 0$ for all $x \neq 0$ and

$$
\arg \alpha_{j}=\inf _{\substack{\operatorname{dim} S \\=j}} \sup _{\substack{x \in S \\ x \neq 0}} \arg (A x, x)=\sup _{\substack{\operatorname{dim} S \\=n-j+1}} \inf _{\substack{x \in S \\ x \neq 0}} \arg (A x, x)
$$

where $S$ ranges over subspaces of $n$-dimensional complex Euclidean space.

Proof. Let $\left(u_{i}\right)$ be an ortho-normal sequence of eigenvectors of $U$ corresponding to $\left(\alpha_{i}\right)$. If $A=X^{*} U X$, then $(A x, x)=\sum_{1}^{n} \alpha_{i}\left|\left(X x, u_{i}\right)\right|^{2}$. If $S$ is the space spanned by $X^{-1} u_{1}, \cdots, X^{-1} u_{j}$, then

$$
\sup _{\substack{x \in S \\ x \neq 0}} \arg (A x, x)=\arg \alpha_{j} \text {. }
$$

Now let $S$ be any subspace of dimension $j$. Let $M$ be the space spanned by $X^{-1} u_{1}, \cdots, X^{-1} u_{n}$. Then there exists a non-zero vector $x$ in $M \cap S$. But

$$
\arg (A x, x) \geqq \inf _{y \neq 0} \arg \sum_{j}^{n} \alpha_{i}\left|\left(y, u_{i}\right)^{*}\right|^{2}=\arg \alpha_{j} .
$$

Therefore

$$
\sup \underset{\substack{x \in S \\ x \neq 0}}{\arg }(A x, x) \geqq \arg \alpha_{j} .
$$

The proof of the second statement is analogous.

Lemma 8 is of course the analogue of the minimax principle for Hermitian matrices. The generalization due to Wielandt [4] also has an analogue for unitary matrices, which we mention without proof since it will not be used.

If $A$ and $U$ satisfy the hypotheses of Lemma 8 and $1 \leqq i_{1}<\cdots$ $<i_{k} \leqq n$, then 


$$
\arg \alpha_{i_{1}}+\cdots+\arg \alpha_{i_{k}}=\inf _{\substack{M_{1} \subset \cdots \subset M_{k} \\ \operatorname{dim} M_{p}=i_{p}}} \sup _{x_{p} \in M_{p}}\left(\arg \beta_{1}+\cdots+\arg \beta_{k}\right)
$$

where $\left(x_{1}, \cdots, x_{k}\right)$ ranges over linearly independent sequences of vectors, and the $\beta_{j}$ are the eigenvalues of the matrix of order $k$ whose $(i, j)$ entry is $\left(U x_{i}, x_{j}\right)$. The number arg $\beta_{1}+\cdots+\arg \beta_{k}$ depends only on the subspace generated by $x_{1}, \cdots, x_{k}$.

LEMMA 9. If $\left(\lambda_{i}\right),\left(\alpha_{i}\right)$ is realizable and $0 \leqq \arg \alpha_{1} \leqq \cdots \leqq \arg \alpha_{n} \leqq \pi$, then $\left(\arg \lambda_{i}\right) \prec\left(\arg \alpha_{i}\right)$.

Proof. By Lemma 1, $\lambda_{i}$ are the eigenvalues of $X^{*} D X$, where $X$ is non-singular and $D=\operatorname{diag}\left(\alpha_{1}, \cdots, \alpha_{n}\right)$. Since the eigenvalues of $X^{*} D X$ vary continuously with the $\alpha_{i}$, we need only prove the theorem for the case where $0<\arg \alpha_{1}$, arg $\alpha_{n}<\pi$. We proceed by induction on $n$. The statement being obvious when $n=1$, suppose $n>1$ and the theorem holds for matrices of order $n-1$. Let $A$ be a triangular matrix with eigenvalues $\lambda_{i}$ which is congruent to $D$. Suppose the $\lambda_{i}$ are arranged so that $\arg \lambda_{1} \leqq \cdots \leqq$ arg $\lambda_{n}$. Let $B$ be the principal minor of $A$ formed from the first $n-1$ rows and columns of $A$. If $x=$ $\left(x_{1}, \cdots, x_{n-1}\right)$ is a vector with $n-1$ components and $y=\left(x_{1}, \cdots, x_{n-1}, 0\right)$ then $(B x, x)=(A y, y)$. Therefore for any such $x \neq 0,(A x, x) \neq 0$ and

$$
0<\arg \alpha_{1} \leqq \arg (A y, y)=\arg (B x, x) \leqq \arg \alpha_{n}<\pi,
$$

by Lemma 8 , since $A$ is congruent to $D$.

By Lemma 7, $B$ is congruent to a unitary matrix $V$. Let the eigenvalues of $V$ be $\beta_{i}$, where arg $\beta_{1} \leqq \cdots \leqq \arg \beta_{n-1}$. Since the quadratic form $(B x, x)$ associated with $B$ is a restriction of the quadratic form associated with $A$, it follows from Lemma 8 that $\arg \alpha_{j+1} \geqq \arg \beta_{j} \geqq \arg \alpha_{j}$, $j=1, \cdots, n-1$. Also by the induction hypothesis $\left(\arg \lambda_{1}, \cdots, \arg \lambda_{n-1}\right) \prec$ $\left(\arg \beta_{1}, \cdots, \arg \beta_{n-1}\right)$. Therefore

$\arg \lambda_{1}+\cdots+\arg \lambda_{r} \geqq \arg \beta_{1}+\cdots+\arg \beta_{r} \geqq \arg \alpha_{1}+\cdots+\arg \alpha_{r}$, $r=1, \cdots, n-1$

and

$$
\begin{aligned}
\arg \alpha_{2}+\cdots+\arg \alpha_{n} & \geqq \arg \lambda_{1}+\cdots+\arg \lambda_{n-1} \\
& \geqq \arg \alpha_{1}+\cdots+\arg \alpha_{n-1}
\end{aligned}
$$

Hence

$$
-\pi<\arg \lambda_{n}-\arg \alpha_{n} \leqq \sum_{1}^{n}\left(\arg \lambda_{i}-\arg \alpha_{i}\right) \leqq \arg \lambda_{n}-\arg \alpha_{1}<\pi .
$$

But

$$
\prod_{1}^{n} \lambda_{i}=|\operatorname{det} X|^{2} \cdot \prod_{1}^{n} \alpha_{i}
$$


Therefore

$$
\sum_{1}^{n} \arg \lambda_{i}=\sum_{1}^{n} \arg \alpha_{i}
$$

The proof is complete.

Lemma 10. If $\left(\beta_{i}\right),\left(\alpha_{i}\right)$ are $n$-tuples of complex numbers of modulus 1 which lie on a line through 0 , and if $(\beta),\left(\alpha_{i}\right)$ is realizable, then $\left(\beta_{i}\right)$ must be a rearrangement of $\left(\alpha_{i}\right)$.

Proof. By Lemma 3 we may suppose that the $\alpha_{i}$ and $\beta_{i}$ are all real. Let $A$ be a matrix with eigenvalues $\beta_{i}$ which is congruent to $\operatorname{diag}\left(\alpha_{1}, \cdots, \alpha_{n}\right)$. Then $A$ is Hermitian and therefore $A$ is also congruent to diag $\left(\beta_{1}, \cdots, \beta_{n}\right)$. But by Lemma 1 it follows that $\left(\alpha_{i}\right),\left(\beta_{i}\right)$ is realizable. Therefore by Lemma 9 we have $\left(\arg \beta_{i}\right) \prec\left(\arg \alpha_{i}\right) \prec\left(\arg \beta_{i}\right)$, from which the present theorem follows immediately.

LEMma 11. Suppose $\left(\beta_{i}\right),\left(\alpha_{i}\right)$ are $n$-tuples of complex numbers of modulus 1 such that $\Pi_{1}^{n} \beta_{i}=\prod_{1}^{n} \alpha_{i}$. Then there exist determinations of $\arg \alpha_{i}, \arg \beta_{i}$ such that

$$
\max \arg \alpha_{i}-\min \arg \alpha_{i} \leqq 2 \pi
$$

and

$$
\left(\arg \beta_{i}\right) \prec\left(\arg \alpha_{i}\right) .
$$

Proof. The statement is obvious for $n=1$. Suppose $n>1$ and it holds for $n$-1-tuples. If any of the $\beta_{i}$ is equal to any of the $\alpha_{i}$, say $\beta_{1}=\alpha_{1}$, then by the induction hypothesis, we can find determinations of the remaining $\arg \alpha_{i}, \arg \beta_{i}$ as stated. If we now choose a value of arg $\alpha_{1}$ which lies between $\mu$ and $\mu+2 \pi$, where $\mu=\min _{i>1} \arg \alpha_{i}$, and set $\arg \beta_{1}=\arg \alpha_{1}$, then the conditions of our theorem will be satisfied, by Lemma 6 . So henceforth we may assume that $\beta_{i} \neq \alpha$, for all $i, j$.

As another special case, suppose the $\alpha_{i}$ are all equal, say to 1 . If we assign arguments to the $\beta_{i}$ such that $0<\arg \beta_{i}<2 \pi$, then $\sum_{1}^{n} \arg \beta_{i}=$ $2 \pi k$, where $k$ is some positive integer $<n$. We need only assign arguments to the $\alpha_{i}$ such that exactly $k$ of them have argument $2 \pi$ and the remaining ones have argument 0 .

Now assume the previous two cases do not occur. The $\alpha_{i}$ divide the unit circle into arcs. At least one of them must contain more than one of the $\beta_{i}$, for if not the $\alpha_{i}$ would be all distinct and each of the $n$ arcs determined by them would contain exactly one of the $\beta_{i}$. We could then assign arguments to arrangements of the $\alpha_{i}, \beta_{i}$ so that

$\arg \alpha_{1}<\arg \beta_{1}<\arg \alpha_{2}<\cdots<\arg \alpha_{n}<\arg \beta_{n}<\arg \alpha_{1}+2 \pi$. 
But then $0<\sum_{1}^{n} \arg \beta_{i}-\sum_{1}^{n} \arg \alpha_{i}<2 \pi$, contradicting the hypothesis $\Pi_{1}^{n} \alpha_{i}=\Pi_{1}^{n} \beta_{i}$.

Let $C$ be an arc containing more than one of the $\beta_{i}$. By changing subscripts, we may assume that the endpoints of $C$ when described counterclockwise are $\alpha_{1}$ and $\alpha_{2}$. Let $\beta_{1}$ be one of the $\beta_{i}$ in $C$ which is nearest to $\alpha_{1}$ and $\beta_{2}$ be one of the $\beta_{i}$ with subscript $\neq 1$ which is nearest to $\alpha_{2}$. Note that $\beta_{1}$ may equal $\beta_{2}$, but $\alpha_{1} \neq \alpha_{2}$. As will be seen from the following argument, we may assume the subarc $\alpha_{1} \beta_{1}$ of $C \leqq$ the subarc $\beta_{2} \alpha_{2}$ of $C$, (all arcs are described counterclockwise). Let $\beta_{1}^{\prime}=\alpha_{1}$ and let $\beta_{2}^{\prime}$ be the point in $\beta_{2} \alpha_{2}$ such that $\beta_{2} \beta_{2}^{\prime}=\alpha_{1} \beta_{1}=\delta$. By the first case of the proof, we may assign arguments to $\beta_{1}^{\prime}, \beta_{2}^{\prime}, \beta_{3}, \cdots, \beta_{n}$ and $\alpha_{1}, \cdots, \alpha_{n}$ so that

and

(1) $\max \arg \alpha_{i}-\min \arg \alpha_{i} \leqq 2 \pi$

(2) $\left(\arg \beta_{1}^{\prime}, \arg \beta_{2}^{\prime}, \arg \beta_{3}, \cdots, \arg \beta_{n}\right) \prec\left(\arg \alpha_{1}, \cdots, \arg \alpha_{n}\right)$.

If $\arg \alpha_{1}$ happens to be the largest of $\arg \alpha_{i}$, and therefore $\arg \alpha_{2}$ is the smallest of arg $\alpha_{i}$, then none of $\beta_{1}^{\prime}, \beta_{2}^{\prime}, \beta_{3}, \cdots, \beta_{n}$ can lie in the interior of $C$. Therefore $\beta_{2}^{\prime}=\alpha_{2}$, and if we decrease $\arg \alpha_{1}$ and $\arg \beta_{1}$ by $2 \pi$, then (1) and (2) will still hold. Thus we may assume $\arg \alpha_{1}<\arg \alpha_{2}$, and therefore $\arg \beta_{1}^{\prime}<\arg \beta_{2}^{\prime}$. Now assign to $\beta_{1}$ the argument $\beta_{1}^{\prime}+\delta$ and to $\beta_{2}$ the argument $\arg \beta_{2}^{\prime}-\delta$. Since

$$
\left(\arg \beta_{1}^{\prime}+\delta, \arg \beta_{2}^{\prime}-\delta\right) \prec\left(\arg \beta_{1}^{\prime}, \arg \beta_{2}^{\prime}\right),
$$

we have by Lemma 6 ,

$$
\begin{aligned}
\left(\arg \beta_{1}, \cdots, \arg \beta_{n}\right) & \prec\left(\arg \beta_{1}^{\prime}, \arg \beta_{2}^{\prime}, \arg \beta_{3}, \cdots, \arg \beta_{n}\right) \\
& \prec\left(\arg \alpha_{1}, \cdots, \arg \alpha_{n}\right) .
\end{aligned}
$$

This completes the proof.

Lemma 12. If $\left(\beta_{i}\right),\left(\alpha_{i}\right)$ are $n$-tuples of complex numbers of modulus 1 which can be assigned arguments such that

$$
\begin{aligned}
& \arg \alpha_{1} \leqq \cdots \leqq \arg \alpha_{n} \leqq \arg \alpha_{1}+2 \pi, \\
& \arg \beta_{1} \leqq \cdots \leqq \arg \beta_{n}, \\
& \left(\arg \beta_{i}\right) \prec\left(\arg \alpha_{i}\right),
\end{aligned}
$$

and

$$
\arg \alpha_{i+1}-\arg \alpha_{i}<\pi, i=1, \cdots, n-1,
$$

then a finite number of pinches will reduce $\left(\alpha_{i}\right)$ to $\left(\beta_{i}\right)$.

Proof. We proceed by induction on $n$. When $n=2$, we have $\arg \alpha_{1} \leqq \arg \beta_{1} \leqq \arg \beta_{2} \leqq \arg \alpha_{2}, \arg \alpha_{1}+\arg \alpha_{2}=\arg \beta_{1}+\arg \beta_{2}$ and $\arg \alpha_{2}-\arg \alpha_{1}<\pi$. Therefore $\arg \beta_{1}-\arg \alpha_{1}=\arg \alpha_{2}-\arg \beta_{2}$ and so 
$\left(\beta_{1}, \beta_{2}\right)$ is a pinch of $\left(\alpha_{1}, \alpha_{2}\right)$.

Suppose $n>2$ and the theorem holds for all $m$-tuples, $m<n$. Let

$$
\delta=\min _{1 \leqq p \leqq n-1} \sum_{1}^{p}\left(\arg \beta_{i}-\arg \alpha_{i}\right) .
$$

There exists $k$ such that $\sum_{1}^{k} \arg \beta_{i}-\sum_{1}^{k} \arg \alpha_{i}=\delta$. It is easy to verify that

$$
\left(\arg \beta_{1}, \cdots, \arg \beta_{k}\right) \prec\left(\arg \alpha_{1}+\delta, \arg \alpha_{2}, \cdots, \arg \alpha_{k}\right)
$$

and

$$
\left(\arg \beta_{k+1}, \cdots, \arg \beta_{n}\right) \prec\left(\arg \alpha_{k+1}, \cdots, \arg \alpha_{n-1}, \arg \alpha_{n}-\delta\right) .
$$

Also

$$
\arg \alpha_{1}+\delta \leqq \arg \beta_{1} \leqq \arg \beta_{n} \leqq \arg \alpha_{n}-\delta .
$$

By the induction hypothesis, we can reduce $\left(\alpha_{1} e^{i \S}, \alpha_{2}, \cdots, \alpha_{k}\right)$ to $\left(\beta_{1}, \cdots, \beta_{k}\right)$ and $\left(\alpha_{k+1}, \cdots, \alpha_{n-1}, \alpha_{n} e^{-i \delta}\right)$ to $\left(\beta_{k+1}, \cdots, \beta_{n}\right)$ by a finite number of pinches. We need only show that $\left(\alpha_{1}, \cdots, \alpha_{n}\right)$ can be reduced to $\left(\alpha_{1} e^{i \delta}, \alpha_{2}, \cdots, \alpha_{n-1}, \alpha_{n} e^{-i \delta}\right)$ by a finite number of pinches. This will follow from the next lemma if we consider only the distinct $\alpha_{i}$.

If the $\alpha_{i}$ all coincide, then so do the $\beta_{i}$ and the statement of our theorem is trivial.

Lemma 13. If $\left(\alpha_{i}\right)$ is an m-tuple of numbers of modulus 1 with assigned arguments such that

$$
\arg \alpha_{1}<\cdots<\arg \alpha_{m} \leqq \arg \alpha_{1}+2 \pi
$$

and

$$
\arg \alpha_{i+1}-\arg \alpha_{i}<\pi, i=1, \cdots, m-1,
$$

and if $\delta$ is a positive number such that $\arg \alpha_{1}+\delta \leqq \arg \alpha_{m}-\delta$, then $\left(\alpha_{i}\right)$ can be reduced to $\left(\alpha_{1} e^{i \delta}, \alpha_{2}, \cdots, \alpha_{m-1}, \alpha_{m} e^{-i \delta}\right)$ by a finite number of pinches.

Proof. This is obvious for $m=2$. Assume $m>2$ and the lemma holds for $m-1$ - tuples. If

$$
\begin{aligned}
& \eta=\min \left(\arg \alpha_{2}-\arg \alpha_{1}, \pi-\left(\arg \alpha_{3}-\arg \alpha_{2}\right), \cdots,\right. \\
& \left.\pi-\left(\arg \alpha_{m}-\arg \alpha_{m-1}\right)\right),
\end{aligned}
$$

and $0<\varepsilon<\eta$, then each sequence in the following list is a pinch of the preceeding sequence:

$$
\alpha_{1}, \cdots, \alpha_{m}
$$




$$
\begin{aligned}
\alpha_{1} e^{i \varepsilon}, & \alpha_{2} e^{-i \varepsilon}, \alpha_{3}, \cdots, \alpha_{m} \\
\alpha_{1} e^{i \varepsilon}, & \alpha_{2}, \alpha_{3} e^{-i \varepsilon}, \cdots, \alpha_{m} \\
\bullet & \bullet \\
\alpha_{1} e^{i \varepsilon}, & \alpha_{2}, \cdots, \alpha_{m-2}, \alpha_{m-1} e^{-i \varepsilon}, \alpha_{m} \\
\alpha_{1} e^{i \varepsilon}, & \alpha_{2}, \cdots, \alpha_{m-1}, \alpha_{m} e^{-i \varepsilon} .
\end{aligned}
$$

Note that $\arg \alpha_{1}+\varepsilon$ need not be $\leqq \arg \alpha_{2}-\varepsilon$, and $\arg \alpha_{2}$ need not be $\leqq \arg \alpha_{3}-\varepsilon$, etc.

We may repeat this cycle of $m$ pinches $k-1$ more times to pass from

$$
\alpha_{1} e^{i \varepsilon}, \alpha_{2}, \cdots, \alpha_{m-1}, \alpha_{m} e^{-i \varepsilon} \text { to } \alpha_{1} e^{k i \varepsilon}, \alpha_{2}, \cdots, \alpha_{m-1}, \alpha_{m} e^{-k i \varepsilon}
$$

as long as $\arg \alpha_{1}+k \varepsilon \leqq \arg \alpha_{2}$, since

$$
\arg \alpha_{2}+p \varepsilon-\arg \alpha_{1}>\arg \alpha_{2}-\arg \alpha_{1}
$$

and

$$
\pi-\left(\arg \alpha_{n}-p \varepsilon-\arg \alpha_{m-1}\right)>\pi-\left(\arg \alpha_{n}-\arg \alpha_{m-1}\right)
$$

for $p<k$. Therefore if $\delta \leqq \arg \alpha_{2}-\arg \alpha_{1}$, we need only choose $\varepsilon=\delta / k$, where $k$ is an integer so large that $\delta / k<\eta$. If $\delta>\arg \alpha_{2}-\arg \alpha_{1}$, choose $\varepsilon=\left(\arg \alpha_{2}-\arg \alpha_{1}\right) / k$, where $k$ is so large that $\varepsilon<\eta$. Then $\left(\alpha_{1}, \cdots, \alpha_{m}\right)$ is reduced to $\left(\alpha_{2}, \alpha_{2}, \cdots, \alpha_{m-1}, \alpha_{m} e^{-i k \varepsilon}\right)$ by the above sequence of pinches. By the induction hypothesis, $\left(\alpha_{2}, \alpha_{3}, \cdots, \alpha_{m-1}, \alpha_{m} e^{-i k \varepsilon}\right)$ can by a finite number of pinches be reduced to $\left(\alpha_{1} e^{i \delta}, \alpha_{3}, \cdots, \alpha_{m-1}, \alpha_{m} e^{-i \delta}\right)$. (The fact that $\alpha_{m} e^{-i k \varepsilon}$ might be equal to one of the $\alpha$, is clearly unimportant.) Therefore $\left(\alpha_{1}, \cdots, \alpha_{m}\right)$ can be reduced to $\left(\alpha_{1} e^{i \delta}, \alpha_{2}, \cdots, \alpha_{m-1}\right.$, $\left.\alpha_{m} e^{-i \delta}\right)$, and the proof is complete.

\section{Proof of Theorem 1 .}

$(2) \rightarrow(1)$ : This is the statement of Lemma 5 .

$(1) \rightarrow(3)$ : If $\left(\lambda_{i}\right),\left(\alpha_{i}\right)$ is realizable, then by Lemma 1 there exists a matrix $A$ and a non-singular matrix $X$ such that $A=X^{*} \operatorname{diag}\left(\alpha_{1}, \cdots, \alpha_{n}\right)$ $X$ and $A$ has eigenvalues $\lambda_{i}$. Therefore $\Pi \lambda_{i}=\Pi \alpha_{i} \cdot|\operatorname{det} X|^{2}$ and hence $\Pi \lambda_{i} /\left|\lambda_{i}\right|=\Pi \alpha_{i}$. If the $\alpha_{i}$ lie on a line through 0 , then $\left(\lambda_{i} /\left|\lambda_{i}\right|\right)$ is a rearrangement of $\left(\alpha_{i}\right)$ by Lemmas 2 and 10 . If the $\alpha_{i}$ lie in a closed half plane through 0 , then by Lemma 3 we may assume they lie in the upper half plane. By Lemma 9 it follows that $\left(\arg \lambda_{i}\right) \prec\left(\arg \alpha_{i}\right)$.

$(3) \rightarrow(2)$ : In case (a), the statement is obvious. In case (c), Lemma 11 and the fact that the $\alpha_{i}$ do not lie in any closed half plane with 0 on its boundary show that the hypotheses of Lemma 12 are satisfied by arrangements of $\left(\lambda_{i}|| \lambda_{i} \mid\right),\left(\alpha_{i}\right)$. In case (b), the hypotheses of 
Lemma 12 also are satisfied by arrangements of $\left(\lambda_{i} /\left|\lambda_{i}\right|\right),\left(\alpha_{i}\right)$. Thus an application of Lemma 12 completes the proof.

\section{REFERENCES}

1. G. H. Hardy, J. E. Littlewood, and G. Polya, Inequalities, Cambridge, 1952.

2. A. Horn, On the eigenvalues of a matrix with prescribed singular values, Proc. Amer. Math. Soc., 5 (1954), 4-7.

3. R. R. Stoll, Linear algebra and mairix theory, New York, 1952.

4. H. Wielandt, An extremum properly of sums of eigenvalues, Proc. Amer. Math. Soc., 6 (1944), 106-110.

UNiversity of CALIFornia, Los ANGELES 


\section{PACIFIC JOURNAL OF MATHEMATICS}

\section{EDITORS}

\section{David Gilbarg}

Stanford University

Stanford, California

\section{R. A. Beaumont}

University of Washington

Seattle 5 , Washington

\author{
A. L. Whiteman
}

University of Southern California Los Angeles 7, California

L. J. Paige

University of California

Los Angeles 24, California

\author{
E. F. BECKENBACH \\ C. E. BURGESS \\ E. HEWITT \\ A. HORN
}

\author{
V. GANAPATHY IYER \\ R. D. JAMES \\ M. S. KNEBELMAN \\ L. NACHBIN
}

ASSOCIATE EDITORS
I. NIVEN

T. G. OSTROM

H. L. ROYDEN

M. M. SCHIFFER
E. G. STRAUS

G. SZEKERES

F. WOLF

K. YOSIDA

\section{SUPPORTING INSTITUTIONS}

\author{
UNIVERSITY OF BRITISH COLUMBIA \\ CALIFORNIA INSTITUTE OF TECHNOLOGY \\ UNIVERSITY OF CALIFORNIA \\ MONTANA STATE UNIVERSITY \\ UNIVERSITY OF NEVADA \\ OREGON STATE COLLEGE \\ UNIVERSITY OF OREGON \\ OSAKA UNIVERSITY \\ UNIVERSITY OF SOUTHERN CALIFORNIA
}

\author{
STANFORD UNIVERSITY \\ UNIVERSITY OF TOKYO \\ UNIVERSITY OF UTAH \\ WASHINGTON STATE COLLEGE \\ UNIVERSITY OF WASHINGTON \\ * * * \\ AMERICAN MATHEMATICAL SOCIETY \\ CALIFORNIA RESEARCH CORPORATION \\ HUGHES AIRCRAFT COMPANY \\ SPACE TECHNOLOGY LABORATORIES
}

Mathematical papers intended for publication in the Pacific Journal of Mathematics should be typewritten (double spaced), and the author should keep a complete copy. Manuscripts may be sent to any one of the four editors. All other communications to the editors should be addressed to the managing editor, L. J. Paige at the University of California, Los Angeles 24, California.

50 reprints per author of each article are furnished free of charge; additional copies may be obtained at cost in multiples of 50 .

The Pacific Journal of Mathematics is published quarterly, in March, June, September, and December. The price per volume (4 numbers) is $\$ 12.00$; single issues, $\$ 3.50$. Back numbers are available. Special price to individual faculty members of supporting institutions and to individual members of the American Mathematical Society: $\$ 4.00$ per volume; single issues, $\$ 1.25$.

Subscriptions, orders for back numbers, and changes of address should be sent to Pacific Journal of Mathematics, 2120 Oxford Street, Berkeley 4, California.

Printed at Kokusai Bunken Insatsusha (International Academic Printing Co., Ltd.), No. 6, 2-chome, Fujimi-cho, Chiyoda-ku, Tokyo, Japan.

PUBLISHED BY PACIFIC JOURNAL OF MATHEMATICS, A NON-PROFIT CORPORATION

The Supporting Institutions listed above contribute to the cost of publication of this Journal, but they are not owners or publishers and have no responsibility for its content or policies. 


\section{Pacific Journal of Mathematics}

\section{Vol. 9, No. $2 \quad$ June, 1959}

Lee William Anderson, On the breadth and co-dimension of a topological lattice

Frank W. Anderson and Robert L. Blair, Characterizations of certain lattices

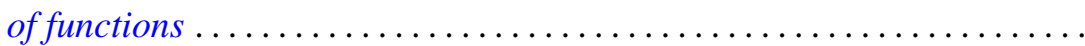

Donald Charles Benson, Extensions of a theorem of Loewner on integral

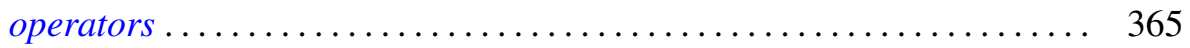

Errett Albert Bishop, A duality theorem for an arbitrary operator ........ 379

Robert McCallum Blumenthal and Ronald Kay Getoor, The asymptotic distribution of the eigenvalues for a class of Markov operators ........

Delmar L. Boyer and Elbert A. Walker, Almost locally pure Abelian

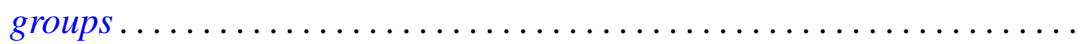

Paul Civin and Bertram Yood, Involutions on Banach algebras ........... Lincoln Kearney Durst, Exceptional real Lehmer sequences .... 415

Eldon Dyer and Allen Lowell Shields, Connectivity of topological

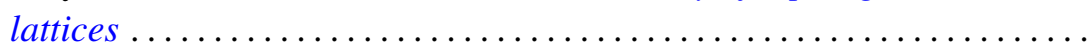

Ronald Kay Getoor, Markov operators and their associated

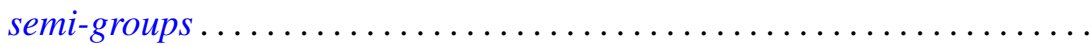

Bernard Greenspan, A bound for the orders of the components of a system of algebraic difference equations

Branko Grünbaum, On some covering and intersection properties in

Minkowski spaces ............................

Bruno Harris, Derivations of Jordan algebras ..............

Henry Berge Helson, Conjugate series in several variables.

Isidore Isaac Hirschman, Jr., A maximal problem in harmonic analysis.

II .

Alfred Horn and Robert Steinberg, Eigenvalues of the unitary part of a matrix

Edith Hirsch Luchins, On strictly semi-simple Banach algebras ...

William D. Munro, Some iterative methods for determining zeros of

functions of a complex variable...

John Rainwater, Spaces whose finest uniformity is metric .

William T. Reid, Variational aspects of generalized convex functions ....

A. Sade, Isomorphisme d'hypergroupoï des isotopes ...... . .

Isadore Manual Singer, The geometric interpretation of a special

connection . . .

Charles Andrew Swanson, Asymptotic perturbation series for characteristic

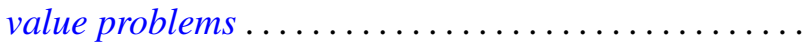

